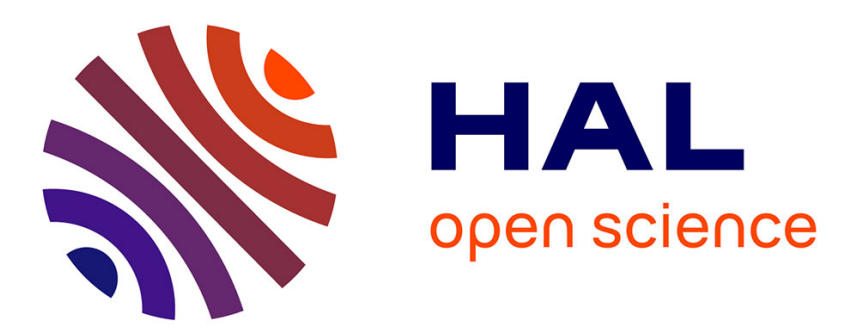

\title{
[Traduction] Fiction utopique et examen critique : la Révolution culturelle dans "L'Âge d'or" de Wang Xiaobo
}

Sebastian Veg, Guillaume Dutournier

\section{To cite this version:}

Sebastian Veg, Guillaume Dutournier. [Traduction] Fiction utopique et examen critique : la Révolution culturelle dans "L'Âge d'or" de Wang Xiaobo. 2007, pp.78 - 91. 10.3406/perch.2007.3575 . halshs02515347

\section{HAL Id: halshs-02515347 \\ https://shs.hal.science/halshs-02515347}

Submitted on 23 Mar 2020

HAL is a multi-disciplinary open access archive for the deposit and dissemination of scientific research documents, whether they are published or not. The documents may come from teaching and research institutions in France or abroad, or from public or private research centers.
L'archive ouverte pluridisciplinaire HAL, est destinée au dépôt et à la diffusion de documents scientifiques de niveau recherche, publiés ou non, émanant des établissements d'enseignement et de recherche français ou étrangers, des laboratoires publics ou privés. 
Fiction utopique et examen critique : la Révolution culturelle dans "L'Âge d'or» de Wang Xiaobo

Sebastian Veg, Guillaume Dutournier

\section{Citer ce document / Cite this document :}

Veg Sebastian, Dutournier Guillaume. Fiction utopique et examen critique : la Révolution culturelle dans «L'Âge d'or» de Wang Xiaobo. In: Perspectives chinoises, n¹01, 2007. pp. 78-91;

doi : https://doi.org/10.3406/perch.2007.3575

https://www.persee.fr/doc/perch_1021-9013_2007_num_101_4_3575

Fichier pdf généré le 29/03/2019 


\title{
Fiction utopique et
}

examen critique

\author{
La Révolution culturelle dans "L’Âge d'or" de Wang Xiaobo (1)
}

SEBASTIAN VEG

La nouvelle qui ouvre la " trilogie des âges » de Wang Xiaobo est aujourd'hui devenue une véritable œuvre culte, notamment parmi les étudiants chinois. Le succès d'un texte qui lie l'envoi à la campagne des " jeunes instruits "à un " âge d'or " de libération sexuelle dans la nature, est certainement en partie imputable à sa dimension scandaleuse ; il recèle toutefois aussi un discours d'une ironie redoutable envers l'utopie agraire maoïste, associée à une régression dans l'animalité. De ce point de vue, Wang Xiaobo se place plutôt du côté d'une réflexion critique, incitant les intellectuels à abandonner les utopies politiques et à s'engager dans la société. Son approche volontiers polémique, rejetant la sacralisation des souffrances passées, a ainsi ouvert un espace indispensable de discussion sur la Révolution culturelle, possible seulement dans le domaine littéraire.

A lors même qu'un examen sérieux de la Révolution culturelle reste impossible dans l'historiographie chinoise ${ }^{(2)}$, la littérature constitue, depuis la fin des années 1970, le seul domaine dans lequel une amorce de réflexion sur la signification des événements de 1966-1976 a pu intégrer la sphère de la discussion publique. Les écrivains ont en effet été les premiers à remettre en question la nécessité, voire la rationalité même des persécutions dont ils furent quasiment tous victimes, et à en appeler à une forme publique de commémoration. Ainsi, Ba Jin (1904-2005) consacre à ce thème bon nombre des essais recueillis dans $A u$ Fil des pensées (Suixiang lu), réclamant dans l'un d'eux la création d'un "Musée de la Révolution culturelle " (1986), et se référant ouvertement au mémorial d'Auschwitz. De même, dans les deux récits qu'elle fait de son envoi dans une "école des cadres du 7 mai ", Six Récits de l'école des cadres (1981) et Chronique des années Bing $W u$ et Ding Wei (1987), Yang Jiang (née en 1911) recourt comme $\mathrm{Ba}$ Jin au détachement ironique pour souligner le caractère irrationnel des événements historiques dans lesquels elle s'est trouvée prise ${ }^{(3)}$. Au même moment émerge le genre fictionnel de la "littérature des cicatrices", que l'on fait remonter habituellement à la publication de "La Cicatrice " (Shanghen) de Lu Xinhua en 1978 ou au récit un peu plus ancien de Liu Xinwu, "Le Professeur principal " (Ban zhuren, 1977), et qui fleurit dans la première moitié des années $1980^{(4)}$. Ce genre, non dénué d'audace à ses débuts, finit pourtant par apparaitre comme insuffisant d'un point de vue esthétique, et cantonné, sur le plan historique, à l'expression plaintive d'une souffrance individuelle, manquant aussi bien de réflexion sur les responsabilités des divers acteurs de la Révolution culturelle, que d'une analyse sérieuse de sa dimension collective et institutionnelle, ainsi que de sa signification historique ${ }^{(5)}$.

Cependant, il faut remarquer que le déclin de ce genre à la fin des années 1980, bien loin de marquer la fin de la littérature sur la Révolution culturelle, s'accompagne d'un regain d'intérêt pour le sujet. À ce moment-là en effet, les cadres ayant survécu aux persécutions (dont ils avaient été les premières victimes) vivent leurs dernières années, tandis que la génération des gardes rouges de 1966 accède au plus haut niveau des responsabilités politiques. Les années 1990

1. Une première version de cet article a été lue lors du colloque « Individu et société dans la littérature chinoise moderne » (Macao, Institut Ricci, 29 novembre 2007), dont nous remercions les organisateurs et auditeurs. Que Michel Bonnin soit également remercié pour sa relecture attentive.

2. Les écrits historiques continuent d'être soumis à la « Résolution concernant certaines questions dans l'histoire du Parti depuis la fondation de la République populaire » (Guanyu jianguo yilai dang de ruogan wenti de jueyi), adoptée le 27 juin 1981 au $6^{e}$ plénum du $11^{\mathrm{e}}$ Comité central. Voir Guo Jian et al., Historical Dictionary of the Cultural Revolution, Lanham, Scarecrow Press, 2006, p. 245.

3. Yang Jiang, Six Récits de l'école des cadres, trad. Isabelle Landry et Zhi Sheng, Paris, C. Bourgois, 1983. Chronique des années Bing Wu et Ding Wei a été traduit sous le titre Sombres nuées, trad. Angel Pino, Paris, C. Bourgois, 1992.

4. L'auteur a lu avec profit l'article de Zhang Yesong, « Dakai shanghen wenxue de lijie kongjian » (Pour ouvrir un espace de compréhension de la littérature des cicatrices), colloque « Individu et société ».

5. II y a bien sûr des exceptions dans les années 1980, comme le récit de Yu Hua, Mille neuf cent quatre-vingt-six (1987) 


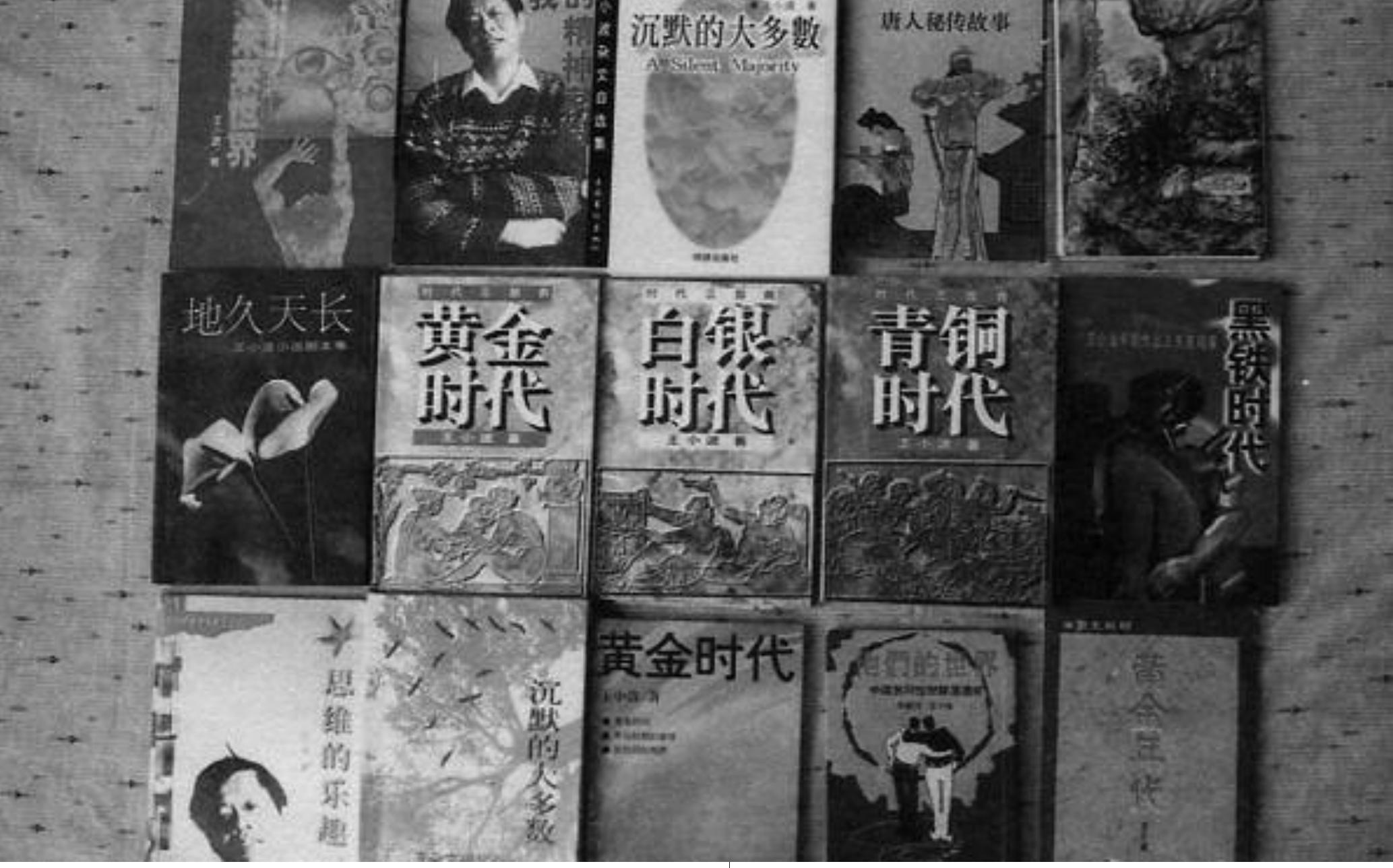

Les ouvrages de Wang Xiaobo ont connu un succès remarquable dans les années 1990

voient ainsi paraitre deux livres qui comptent sans doute parmi les œuvres fictionnelles les plus réfléchies et les plus provocantes sur la période : «L'Âge d'or » de Wang Xiaobo (Huangjin shidai, 1992) et Le Livre d'un homme seul de Gao Xingïian (Yige ren de shengjïng, 1998) ${ }^{(6)}$. Il est à noter que Gao Xingjian, né en 1940, est l'un des plus jeunes membres de la génération des cadres persécutés et envoyés dans les écoles du 7 mai, alors que Wang Xiaobo (19521997) appartient à la génération suivante, celle des « jeunes instruits " (zhiqing), ce qui lui vaut de passer trois ans (1968-1970) dans une ferme collective du Yunnan, puis d'être transféré dans le Shandong avant de retourner finalement à Pékin en 1972, où il devient ouvrier dans une usine pour encore six ans. Mais tandis que le livre de Gao Xingjian est seulement publié à Hong Kong et Taiwan, ce qui le rend peut-être moins central pour comprendre la façon dont se construit la mémoire de la Révolution culturelle dans la Chine d'aujourd'hui ${ }^{(7)}$, la nouvelle de Wang Xiaobo (d'abord publiée en 1992) ainsi que la Trilogie des âges dont elle fait partie (la nouvelle constituant le cœur du premier volume de la trilogie, lui aussi intitulé L'Âge d'or) sont devenues des œuvres culte, lues par les intellectuels et les étudiants sur tous les campus de Chine. Wang Xiaobo reçut en 1993 le prix du Lianhebao ${ }^{(8)}$ à Taiwan, et se retira de l'enseignement universitaire pour devenir écrivain à plein temps - bien que toujours inconnu en Occident -, jusqu'à sa mort brutale d'une crise cardiaque en $1997^{(9)}$.

Les œuvres de Wang Xiaobo touchent à la politique chinoise (la Révolution culturelle dans L'Âge d'or, l'unité de travail, la manipulation de l'opinion et de l'art) et se montrent souvent hardies dans l'expression de la sexualité : deux aspects qui les rendirent scandaleuses même aux yeux des étudiants et des intellectuels blasés de la Chine des années 1990. Le récit qui nous occupe doit ainsi en partie son succès en Chine au lien paradoxal et ironique qu'll établit entre l'envoi des " jeunes instruits " à la campagne et un "âge d'or » de libération sexuelle en pleine nature. Pourtant, audelà du scandale et du mythe personnel de la mort prématu-

6. On pourrait citer d'autres œuvres des années 1990, comme « La cruauté des animaux » (Dongwu xiongmeng, 1993) de Wang Shuo, adapté au cinéma par Jiang Wen dans Yangguang canlan de rizi (Des Jours éblouissants, 1994) ; "L'ère du tatouage » (Ciqing shidai, 1993) de Su Tong ; "Poèmes à l'idiot » (Shagua de shipian, 2000) de Ge Fei. Mais aucune de ces œuvres n'a le statut d'œuvre culte dont jouit la nouvelle de Wang Xiaobo.

7. Cf. S. Veg, «Fuite sans fin et exil impossible : Le Livre d'un homme seul de Gao Xingjian », colloque " Exils en France au XXe siècle », Cerisy-la-Salle, 14-21 août 2006.

8. Ce prix témoigne également du succès considérable dont Wang jouit à Taiwan, et ce bien que son œuvre soit étroitement liée à l'histoire et à la politique chinoise.

9. Nous résumons les éléments biographiques présentés dans le catalogue de l'exposition organisée par le Musée Lu Xun de Pékin en 2005 : Li Yinhe, Zheng Hongxia (éd.), Yige teli duxing de ren (Un homme indépendant et solitaire), Pékin, Dazhong wenyi, 2005. 
rée de l'écrivain, les lecteurs chinois ont certainement été attirés, jusquà aujourd'hui, par l'ambition qu'a Wang de se confronter à la Révolution culturelle. Or, l'histoire de celle-ci n'est pas tant racontée au lecteur en une suite d'événements, que condensée dans un espace-temps utopique et presque fantastique, lui-même placé à son tour dans le contexte d'une évolution beaucoup plus vaste, que Wang formule en termes quasi cosmiques dans sa Trilogie des âges. La Révolution culturelle, qui correspond à l'" âge d'or ", trouve ainsi sa place entre l'" âge de bronze " des récits fantastiques dans le style des chuanqi Tang, lesquels explorent les mœurs sexuelles et politiques de la Chine classique, et l'« âge d'argent » dystopique du « Monde à venir ", où les individus subissent un contrôle étatique toujours plus efficace et imperceptible. De cette façon, ce qui pourrait apparaitre comme une anomalie historique (et fait bien l'objet d'une description qui ne minimise en rien la violence et l'absurdité de la période) trouve finalement une place - quoique largement ironique et critique - à l'intérieur d'un schéma historique plus large, dans lequel l'individu se voit régulièrement opprimé par des structures de pouvoir toujours changeantes.

L'interprétation la plus commune de cette surprenante construction fictionnelle consiste à dire que si toutes les formes d'organisation sociale sont intrinsèquement répressives (quoique différemment selon les époques), durant la Révolution culturelle, le " retour à la nature » des jeunes instruits fait en revanche surgir, libérée des contraintes sociales et comme par une ruse de l'histoire, une sorte de force primale incarnée par la sexualité. Selon cette lecture, la puissance sexuelle de Wang Er, personnage principal de "L'Âge d'or », est en elle-même anti-autoritaire, et représente donc une forme de résistance à l'oppression et à l'omniprésence de l'État (maoiste). Le critique Ding Dong, en particulier, a développé l'idée selon laquelle le sexe constituerait dans l'œuvre de Wang Xiaobo « l'ultime défense de l'individu contre le monde extérieur ${ }^{(10)}$ ", allant jusqu'à dire que la " laideur " du " petit bonze ${ }^{(I I)}$ » de Wang Er représente le pendant symétrique des mensonges propagés par le pouvoir politique. Cette interprétation apparait quelque peu problématique, dans la mesure où la rébellion du personnage ne peut à la fois révéler le potentiel anti-autoritaire d'une sexualité débridée et exprimer une révolte individuelle contre un système politique : la sexualité de Wang Er, si elle est libre de toute contrainte, est également tout sauf réfléchie, c'est un simple état de fait sur lequel Wang Er semble lui-même avoir peu de contrôle.

Dans le récit de Wang Xiaobo, ces tendances divergentes coexistent : d'un côté, un esprit critique et une ironie individuelle, de l'autre, une régression vers un état de nature utopique, dans lequel les humains vivent véritablement comme des buffles du Yunnan qui n'auraient pas été castrés par le chef de l'équipe de production. Cette tension est peutêtre encore plus présente dans l'idée d'" âge d'or ", laquelle peut être comprise soit comme une ère mythique précédant toute histoire et toute organisation sociale, soit à l'inverse comme la peinture mordante de la misère de l'" Amour au temps de la révolution " - en écho à la libération des passions sexuelles dans une autre situation sociale extrême décrite dans le roman de Gabriel Garcia Marquez. En ce sens, tout porteur qu'il est de la dimension utopique d'une sexualité libérée des conventions sociales, l'âge d'or que dépeint Wang Xiaobo résonne aussi, de façon fortement ironique, avec l'utopie agraire maoiste qui devait se réaliser avec l'envoi des intellectuels aux champs. Certes, l'« âge d'or » envisagé par Mao ne correspond sans doute pas au type de vie que Wang Er et Chen Qingyang finissent par mener dans le Yunnan. Cependant, celle-ci comporte une part de ce que certains ont voulu voir dans les théories maoistes : la défiance radicale à l'égard de toute structure de pouvoir. Pour toutes ces raisons, les récits de Wang Xiaobo demeurent ambigus, et avares d'indices sur la façon dont il convient d'interpréter cet âge d'or qui marque le vingt-et-unième anniversaire de Wang Er : doit-il être compris comme une accusation portée contre une humanité qui ne s'accomplirait qu'en renonçant à son humanité, ou au contraire comme une façon de célébrer l'affranchissement d'une soumission masochiste à la politique paradoxale conséquence de la politique frondeuse du maoïsme totalitaire ? Cet article tente d'examiner et de clarifier la façon dont, selon Wang Xiaobo, l'individu peut s'émanciper de l'étouffante pression politique et sociale, et dans quelle mesure la Révolution culturelle peut être vue comme une occasion paradoxale pour l'individu d'échapper à une telle oppression.

\section{Nature libératrice, société castratrice}

"L'Âge d'or " contient sans conteste un discours dans lequel le séjour à la campagne du " jeune instruit " Wang Er apparait comme une libération paradoxale des contraintes de la vie sociale, un thème classique de la littérature des zhiqing $^{(12)}$. Nombreux sont en effet les zhiqing qui, soustraits

10. Cité par Wendy Larson, dans "L'indifférence, les intellectuels, le sexe et le temps dans L'Âge d'or de Wang Xiaobo », in Annie Curien (éd.), Écrire au présent. Débats littéraires franco-chinois, Paris, Éd. de la MSH, 2004, p. 201-230 (p. 216, note 15).

11. Ici comme dans le reste de l'article, nous nous référons à l'édition française : L'Âge d'or, trad. Jacques Seurre, Versailles, Éditions du Sorgho, 2001 (désormais abrégé $A O$ ). Les citations sont parfois légèrement modifiées en fonction de Wang Xiaobo, Huangjin shidai, Guangzhou, Huacheng, 1997.

12. Voir par exemple Michel Bonnin, Génération perdue, Paris, Presses de l'EHESS, p. 2223, et la préface de Michel Bonnin « L'Âge d'or du petit bonze » à L'Âge d'or de Wang Xiaobo, traduction Jacques Seurre, Versailles, Éd. du Sorgho, 2001, p. 18. 
au contrôle de leurs parents et de leurs professeurs et placés dans un milieu rural qui les condamnait souvent à la promiscuité, ont connu une forme paradoxale de liberté : le rigorisme maoiste régnant dans les zones urbaines était en effet appliqué ou respecté de façon moins sévère dans le milieu paysan où ils vivaient. Pourtant, si ce thème relève d'ordinaire d'une veine sentimentale qui le rattache aux souvenirs tendres de l'âge adolescent ou post-adolescent, Wang Xiaobo, tournant le dos à toute sentimentalité, lui donne une forme explicitement sexuelle.

Le titre "L'Âge d'or " se comprend d'abord en référence à l'extraordinaire vitalité physique dont le personnage principal fait l'expérience, lors de son vingt-et-unième anniversaire, à travers une érection aux dimensions monumentales sur laquelle s'ouvre le texte :

Le jour de mes vingt-et-un ans, je gardais les buffles au bord de la rivière. C'était l'après-midi, je dormais couché sur la prairie. Avant de m'endormir, je m'étais recouvert le corps de feuilles de bananier. Lorsque je me réveillai, celles-ci avaient toutes disparu [...]. Sous le soleil tropical de la saison sèche, tout mon corps était devenu écarlate, en proie à des démangeaisons insupportables. Mon petit bonze se dressait tout droit face au ciel, il avait gagné des dimensions jamais atteintes. [...] Parmi les innombrables érections que j'avais eues dans ma vie, aucune n'avait atteint celle-ci en puissance. Peut-être la solitude du lieu y était-elle pour quelque chose; il n'y avait pas âme qui vive alentour. $(A O$, p. 29)

C'est un passage hautement symbolique, situé le jour de la majorité de Wang Er, dans une sorte d'utopie bucolique, le personnage vivant parmi les animaux, vêtu seulement de feuilles de bananier. Son corps réagissant à une stimulation cosmique (rayons du soleil et caresses du vent), l'érection atteint elle aussi à des dimensions cosmiques - phénomène que Wang Er explique par le fait qu'il n'a jamais été aussi loin de toute présence humaine. Dès lors, le désir sexuel apparait comme quelque chose de naturel et d'inné, indissociable de la croissance d'un homme, mais d'ordinaire réprimé par la présence des autres, par le conformisme des discours et pratiques sociales.

Au cours du récit, le sexe se retrouve fréquemment associé à cette dimension cosmique, à une sorte de communion entre le corps de Wang Er et les éléments. Symétriquement au thème du soleil, la scène du premier rapport sexuel de Wang Er et Chen Qingyang est baignée par le clair de lune :
Ce soir-là, le soir de mes vingt-et-un ans, je cessais d'être un puceau. J'avais réussi à séduire Chen Qingyang, à l'attirer dans la montagne. La lune ne s'était montrée que peu de temps, elle avait laissé la place aux étoiles, innombrables comme les gouttes de rosée du matin. Il n'y avait pas de vent, la montagne était silencieuse, j'avais fait l'amour avec Chen Qingyang, j'étais un homme. ( $A O$, p. 42)

Dans ce passage également, c'est bien plus une nécessité d'ordre biologique (l'âge adulte) et un environnement cosmique que l'amour ou les sentiments qui viennent expliquer, pour Wang Er, la perte de sa virginité. La scène se répète maintes fois, comme par exemple au chapitre 7, où Wang Er et Chen Qingyang font l'amour dans les brumes de l'aube sous le regard d'un buffle blanc $(A O$, p. 90$)$, sur un lopin de terre si fertile qu'il grouille de vers et sur lequel Wang Er éjacule, si bien que Chen Qingyang demande : " Est-ce qu'on pourrait faire pousser en terre un petit Wang $\mathrm{Er} ?$ ? $(A O$, p. 99)

Cette libération du corps dans la nature contraste avec l'image de structures sociales castratrices et répressives. Les buffles que Wang Er est censé conduire au moment où il s'endort ont été castrés par le chef de l'équipe de production pour les rendre dociles:

Pour les buffles ordinaires, le couteau suffisait. Mais pour ceux que la nature avait particulièrement avantagés, il fallait utiliser la technique du maillet, c'est-à-dire inciser le scrotum, en sortir les testicules et les faire éclater d'un seul coup de maillet. La vie de l'animal qui avait subi tout cela était réduite désormais à la rumination, et quand on finissait par les abattre, il n'y avait même pas besoin de les attacher. Le chef de l'équipe de production, qui dans ces occasions tenait le maillet, ne doutait pas qu'une telle opération, pratiquée sur des êtres humains, ait sur eux des effets similaires. $\dot{A}$ chaque fois, il nous criait : "Tas de gros buffles sauvages! Il faudrait vous les écraser d'un bon coup de maillet pour vous faire marcher droit! "Selon sa façon de voir, cette chose rouge vif qui faisait partie de mon corps, si opiniâtrement dressée, qui avait un pied de long, c'était l'incarnation du mal. (AO, p. 30)

Ce passage permet d'établir un lien profond entre la vitalité, la force et la volonté de vivre, et l'expression biologique d'une sexualité sans entraves. La volonté de vivre des buffles est irrémédiablement brisée quand le chef de l'équipe de production, en un geste symbolique, écrase leurs testicules 
Wang Xiaobo à l'Université de Pittsburgh, dans les années 1980 (DR) avec un marteau (sans que cela dépende d'une quelconque nécessité médicale). Par contraste, le désir sexuel irrépressible de Wang Er représente une énergie rebelle, défiant toute forme de contrôle.

Les préservatifs sont un autre symbole de la façon, dans ce cas plus progressive, dont les pressions sociales étouffent les forces de la nature. Chen Qingyang, qui représente au début une forme de conformisme social, résiste aux avances de Wang Er la première nuit en essayant de lui faire porter un préservatif ("J'étais au comble de l'excitation, si bien que je ne fus guère affecté par la brusquerie de ses manières. Après avoir mis le préservatif, je grimpai à nouveau sur elle, mais j'étais trop haletant et fébrile pour arriver à mes fins "; $A O$, p. 39-40). Seule exception à cette règle : les préservatifs "cent pour cent caoutchouc naturel " que Wang et Chen utilisent au cours de leur idylle bucolique dans le moulin du père Liu. Ces derniers, quoique peu appréciés de la population locale parce qu'ils «obstru[ent] les échanges entre le yin et le yang, ce qui [a] à la longue un effet débilitant ", sont néanmoins " les meilleurs que j’eusse jamais utilisés " $(A O$, p. 73). Mais à partir du moment où Chen Qingyang se trouve vraiment impliquée dans leur entreprise de retraite en pleine nature, c'est elle-même qui ne veut plus en utiliser :

Elle ne voulait pas que je mette "cette horrible chose ", elle voulait, disait-elle, me faire une nichée de marmots, jusqu'à en avoir les seins " comme ça ", au bout de quelques années, dit-elle en tirant le bout de ses seins vers le bas pour qu'ils aient l'air de pendre. Je trouvais ça vraiment disgracieux, et lui dis : "Oui, bon, on verra ça; mais ça me gênerait que tu aies les seins qui pendent. "Ce qui fait qu'en définitive jai mis un préservatif. Par la suite, elle perdit tout intérêt pour ces choses-là. ${ }^{(13)}(A O$, p. 78)

Cette étape marque une rupture radicale avec les conventions sociales, entraînant la transformation de Chen Qingyang en " koala " grimpant sur Wang $\operatorname{Er}(A O, 76)$. Wang Xiaobo recourt également au cliché des régions à " minorités ", où les coutumes sexuelles sont réputées plus "libérées ", ce que suggère l'image de Chen Qingyang "s'indigénisant " quand elle porte un pagne Dai, et de Wang Er songeant à un mariage dans une famille Achang, dans laquelle ils contribueraient à des activités de procréation collective avec " grande sœur Achang " et " grand frère Achang " $(A O, 102)$. Néanmoins, comme Wang Xiaobo le savait sans doute, cette idée de promiscuité sexuelle n'est rien

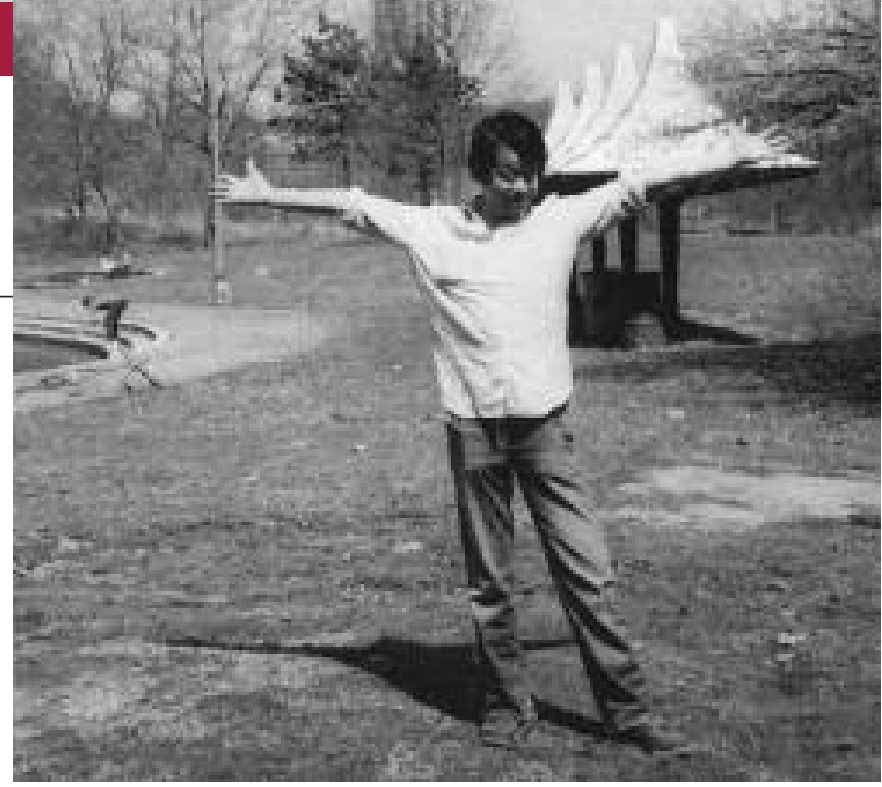

d'autre qu'un cliché de l'" orientalisme intérieur " (D. Gladney $)^{(14)}$, et Wang Er et Chen Qingyang sont en fait loin de faire partie de la société locale.

Comment donc interpréter cette incompatibilité apparente entre les niveaux individuel et socio-politique ? Beaucoup des premiers commentateurs de Wang Xiaobo situent ses œuvres dans la veine de Foucault ou de Marcuse, y distinguant des individus soumis à une forme toujours changeante de castration opérée par la société en général. L'utopie rurale de "L'Âge d'or " leur apparait comme un moment unique au cours duquel, dans un contexte historique exceptionnel, l'individu peut rejeter la forme masochiste de servitude qui le lie aux structures sociales, et vivre selon des instincts essentiellement sexuels. Sans que l'on sache clairement si Wang a effectivement lu des traductions de Foucault, on peut en effet présumer que, pendant ses études aux États-Unis (1984-1988), il a acquis au moins une connaissance de seconde main de L'Histoire de la sexualité( ${ }^{(15)}$. Il nous semble pourtant qu'une telle interprétation, tout en coincidant avec quelques-unes des lectures et positions théoriques de Wang Xiaobo, ne rend pas vraiment justice à la structure de l'ouvrage.

Certes, Wang Er rompt doublement avec la société, d'abord en étant envoyé dans le Yunnan comme jeune instruit, ensuite en s'enfuyant de son équipe de production à deux re-

13. On trouve des discussions semblables sur la nature castratrice des préservatifs dans "Sanshi er li » (À trente ans, on se tient debout), L'Âge d'or, p. 98 ; "Sishui liunian » (Comme le temps passe), ibid., p. 153, en particulier dans ce dernier extrait.

14. Voir Dru C. Gladney, "Representing Nationality in China: Refiguring Majority/Minority Identities ", The Journal of Asian Studies, vol. 53, n 1, février 1994, p. 92-123.

15. Après avoir obtenu un Master of Arts à l'université de Pittsburgh, Wang Xiaobo retourne en Chine comme professeur de sociologie à l'université de Pékin en 1988. Les trois volumes de l'Histoire de la sexualité de Foucault ont été publiés en français entre 1976 et 1984. L'une des premières traductions chinoises de Foucault est le volume 1 de cette œuvre, traduit en 1988-1989 : Mixie'er Fuke, Xingshi, trad. Huang Yongming, Yu Banfa, Shanghai, Wenhua, 1988, 132 p. (1 ${ }^{\mathrm{e}}$ partie $)$; et trad. Zhang Tingshen, Shanghai kexue jishu wenxian, 1989, 429 p. (2 partie). La veuve de Wang Xiaobo, Li Yinhe, en a publié un commentaire, sous le titre Foucault et la sexualité, qui a eu également une certaine influence en Chine : Fuke yu xing : jiedu Fuke "Xingshi », Jinan, Shandong renmin, 2001. Li Yinhe mentionne aussi fréquemment l'influence de l'éthologiste américain Alfred Kinsey (1894-1956), auteur du « rapport Kinsey » sur le comportement sexuel et initiateur de la sexologie, sur ses propres écrits et sur ceux de Wang Xiaobo. 
prises : une première fois après avoir reçu des coups de tabouret sur le dos lors d'un "réunion de critique et de lutte" (pidouhui), une deuxième fois après avoir été "lutté " par un député de l'armée qui préconise une rééducation idéologique immédiate. Il achète alors un fusil de chasse à double canon et disparait avec Chen Qingyang dans les montagnes voisines de la frontière birmane, où ils vivent en ermites pendant six mois (chapitres 3, 4, 5 et 8).

Mais s'il est vrai que cette vie dans la nature peut être considérée comme une forme radicale de fuite loin des contraintes sociales, elle n'est nullement idéalisée. La sexualité peut certes apparaître en elle-même comme une forme de résistance à la politique révolutionnaire, ou même comme l'ultime défense d'un individu contre une politique par trop envahissante, mais il s'agit néanmoins de la résistance la plus simple, la moins consciente et la moins critique : quand Chen et Wang Er font l'amour dans les montagnes parmi les brumes de l'aube sous le regard d'un buffle blanc, ce n'est pas par plaisir, ni pour marquer leur liberté par rapport à la pruderie maoiste (comme lors de leur première rencontre sur la montagne), mais seulement par instinct de survie :

Une heure après, nous fûmes réveillés par le froid. Les trois couches de couverture étaient complètement trempées et le feu de bouse éteint. [...] Elle se mit à ramper vers moi : "Ça ne va pas, dit-elle, si ça continue nous serons malades tous les deux. Il faut faire l'amour, tout de suite. " $(A O$, p. 89)

Tandis que, dans d'autres ouvrages rattachant la Révolution culturelle à une sexualité exacerbée (La Moitié de l'homme, c'est la femme de Zhang Xianliang, Le Livre d'un homme seul de Gao Xingjian et, plus récemment, Servir le peuple de Yan Lianke), le sexe apparait comme une forme de transgression qui en définitive offre à l'individu un espace de résistance minimal face aux événements historiques, Wang Xiaobo en revanche le présente dans ce passage comme une forme de régression vers une existence animale, un besoin relevant d'une pure nécessité biologique (conserver la chaleur pour survivre), entièrement dénué de sentimentalisme, et des sentiments humains ou humanistes qui caractérisent la "littérature des cicatrices" (par exemple dans le roman de Zhang Xianlang). Loin d'idéaliser cette vie, le narrateur s'étend longuement sur les différentes formes d'épreuves (par exemple l'utilisation du fumier de bétail) qu'ils doivent endurer. Ainsi, Wang Er insiste sur le fait que Chen le trouve "non civilisé ", et note : "C'est pour cela, entre autres, qu'elle ne m'aimait pas, qu'elle ne me trouvait même pas sympathique " (AO, p. 87). Pour toutes ces raisons, il est sans doute important de lire ce texte sans le limiter à une libération foucaldienne de l'individu, délivré de sa soumission masochiste aux conventions sociales puritaines ou sadiques grâce au biais inattendu du maoïsme. L'idée d'un "âge d'or " ne doit donc pas être prise au pied de la lettre, mais être interprétée dans le contexte historique de la Révolution culturelle et plus généralement de la société maoiste et postmaoiste.

\section{L'âge d'or de la politique utopique}

Les commentateurs ont souvent sous-estimé la dimension hautement ironique de la notion d' " âge d'or " elle-même, qui peut être vue comme une parodie de l'utopie maoïste et de sa promesse d'un âge d'or du retour à une société rurale basée sur le communisme agricole. Wang Xiaobo tourne cette utopie en dérision en faisant du Yunnan le laboratoire d'une expérience sexuelle cosmique au sein de la nature. De ce point de vue, le motif de la fuite hors de l'équipe de production apparait, à l'intérieur de la structure narrative, comme une forme de duplication ironique de la sortie imposée (quoique officiellement "volontaire ") de la société urbaine.

Les réalités de la Révolution culturelle, qui prennent la forme d'une série ininterrompue d'humiliations (comme par exemple nourrir les porcs) et d'actes de violence, ne sont nullement absentes du récit de Wang Xiaobo ${ }^{(16)}$. Wang Er a été envoyé dans une équipe de production agricole dans le Yunnan afin de subir une rééducation par les paysans "pauvres et moyens-pauvres ". Il y fait l'expérience de la réaffirmation du contrôle de l'armée sur la ferme, avec l'apparition d'un "député militaire " qui annonce ensuite la transformation de la ferme d'État en une ferme militaire ( $A O$, p. 68), puis l'arrivée d'un grand nombre de cadres de l'armée. Coordonnés par le Bureau de la sécurité publique (Ren bao zu), contrôle et dénonciations sont monnaie cou-

16. De la même façon, le suicide de M. He joue un rôle central dans « Sishui liunian » (Comme le temps passe), dans le même recueil. 
rante. Presque immédiatement après la scène de l'érection monumentale de Wang Er au bord de la rivière, une violente bagarre se déclenche entre jeunes instruits et jeunes paysans, laquelle entraine la convocation d'une "réunion de lutte" (douzhenghui) par le chef d'équipe : Wang Er y est battu avec un tabouret par la mère d'un jeune paysan, ce qui le paralyse pour un temps. Au chapitre 9, il participe à une " excursion de lutte " (chu douzheng chai) à la frontière birmane :

Notre charrette était pleine de contre-révolutionnaires historiques, de voleurs, de partisans de la voie capitaliste, de " chaussures trouées", etc. Les personnes considérées comme faisant partie des " contradictions antagonistes " côtoyaient celles relevant des " contradictions au sein du peuple ". Après les avoir fait travailler, on les transportait à la frontière leur faire subir des séances publiques d'accusation, afin de " consolider politiquement la défense des frontières ». (AO, p. 109-110)

Le narrateur souligne l'absurdité qui règne à tous les niveaux dans cette expédition : une équipe hétéroclite d'individus est arbitrairement étiquetée comme criminelle et répartie dans les deux catégories maoïstes des ennemis du peuple et des " contradictions au sein du peuple ». Le groupe se voit jeté sur un tracteur pour représenter un "spectacle de lutte " (douzheng yi tai) à destination des gardes birmans (et potentiellement des espions nationalistes qui sont supposés les avoir infiltrés) de l'autre côté de la frontière ${ }^{(17)}$, avec la justification du mot d'ordre " consolider politiquement la défense des frontières " qui porte l'absurde à son comble. Il s'avère heureusement que Chen et Wang sont seulement des " contradictions au sein du peuple " (c'est-à-dire non antagonistes; $A O$, p. 114), ce qui ne les préserve cependant pas de la " colère des masses".

De même, c'est pour le bénéfice du même groupe improbable qu'une "réunion de critique et de lutte " (pidouhui) se tient au chapitre 6 ; Wang et Chen sont " fortement encouragés » à y participer.

Après tout, nous dirent-ils, une petite réunion de lutte ne pouvait pas nous faire de mal, au contraire, ça vous stimule l'esprit, et après on ne commet plus de faute. Avec tant d'avantages, comment pouvait-on rechigner? Le jour de la réunion arriva. Il y avait plusieurs milliers de personnes, venues de la ferme d'État ou des brigades de production avoisinantes. Chen et moi nous tenions debout, parmi beaucoup d'autres, sur l'estrade. Notre problème avait été défini ainsi :
"Avec une pensée malsaine et des mœurs dissolues, ils se sont enfuis dans la montagne pour échapper à leur réforme ; mais finalement, sous l'influence $d u$ Parti, ils sont sortis de l'ombre et sont revenus d'euxmêmes à la lumière. » $(A O$, p. 80$)$

Bien que d'une grande économie, l'ironie ne perd rien de son mordant : parler des " avantages " que représente le pidouhui, ainsi que de la "lumière " de la politique du Parti qui a finalement permis aux pécheurs de descendre de la montagne, revient à citer le discours officiel sur un ton facétieux, pour évidemment le subvertir totalement.

En réalité, le déroulement de ces réunions interminables est décrit comme une représentation théâtrale, raillée dans des termes rappelant l'observation anthropologique des fêtes des " minorités »:

\section{[...] lutter les " chaussures trouées " était devenu} une espèce d'activité récréative traditionnelle. Quand tout le monde était fatigué par les travaux des champs, le chef d'équipe disait : "Ce soir, on va se distraire un peu, on va lutter les "chaussures trouées" ». [...] Par la suite, un grand nombre de cadres militaires vinrent gérer la ferme d'État et ordonnèrent que l'on mette fin à ces pratiques, sous prétexte qu'elles manquaient de consistance politique. Puis ce fut le retour de "l'édification en commun par l'armée et le peuple ", et les réunions pour lutter les "chaussures trouées » recommencèrent. Le chef de la Ligue nous commanda de retourner à l'équipe de propagande et de nous préparer aux réunions à venir. $(A O, \text { p. 114 })^{(18)}$

La critique des "problèmes de style [de vie] " (zuofeng wenti) et des " chaussures trouées " (poxie, terme censé désigner les femmes " de vie légère ") devient partie intégrante d'une forme de cirque politique, dans lequel les décisions sont prises et révisées à loisir. La représentation théâtrale elle-même est bien sûr une forme d'humiliation publique planifiée et calibrée avec précision, et la distance affirmée du narrateur ainsi que sa position ironique montrent qu'il refuse de se laisser humilier par les démonstrations publiques de son prétendu manque de moralité. Bien au contraire, en affirmant que ces humiliations publiques excitent sexuellement

17. Voir M. Bonnin, «L'Âge d'or du petit bonze », art. cit., p. 10.

18. Nous modifions, dans tout ce passage, la traduction pour mettre en valeur la construction transitive du verbe douzheng, caractéristique de l'époque de la Révolution culturelle, que nous traduisons donc par « lutter », plutôt que par « faire un meeting contre ». 

L'euphémisme hypocrite se combine avec l'avidité du commissaire pour les détails obscènes, lorsque Wang Er emploie métaphoriquement l'expression "renforcer la grande amitié " pour dire "f faire l'amour ", et que les chefs exigent plus de détails : "Les dirigeants voulaient que je précise qui était ce Coït, qu'est-ce que nous entendions à la fin par "renforcer la grande amitié" et comment pouvait-on la renforcer à l'envers ou à l'endroit ? " $(A O$, p. 98) Les idéaux élevés de la politique révolutionnaire sont ainsi démystifiés, n'apparaissant plus que comme le moyen de satisfaire des penchants voyeuristes : les chefs du Bureau de la Sécurité publique, tout en soutenant que Wang Er a besoin d'une « rééducation idéologique ", sont eux-mêmes uniquement intéressés par les détails sexuels de ses fautes. Ils commencent par dire à Wang Er que le contenu de ses aveux n'importe pas vraiment : " Alors avouez au moins que vous êtes des traitres qui êtes passés à l'ennemi ; il faut bien avouer quelque chose, tout de même! Vous n'avez qu'à vous mettre d'accord entre vous. Si tu n'avoues rien, on ne te relâchera pas " $(A O, \mathrm{p}$. 75). Ainsi, tout le système politique semble destiné à perdurer uniquement grâce à la production d'une forme de fiction. Mais rapidement, les chefs prennent plaisir aux descriptions de la liaison amoureuse, et continuent à leur en faire écrire après la réunion de lutte : "Après cette séance, on nous laissa à peu près en paix, mais il fallut continuer à rédiger des autocritiques, les dirigeants de la Ligue des jeunes communistes voulaient les lire " $(A O$, p. 80$)$. Pour ces raisons, le lecteur finit par se demander si Wang Er n'a pas en fait introduit la notion de "grande amitié " dans sa confession pour tourner secrètement en dérision les chefs qui le forcent à l'écrire. On peut se demander en outre dans quelle mesure Wang Xiaobo n'a pas également eu l'intention de faire de la curiosité des commissaires un symbole du voyeurisme des lecteurs à venir qui, captivés par la dimension scandaleuse du texte, risqueraient de manquer son aspect de critique politique.

Wang Er est en réalité tout à fait conscient de s'engager dans une forme d'activité littéraire, sinon fictionnelle, comme en témoigne le fait qu'il présente le manuscrit à un diplômé en littérature américaine, qui à son tour l'évalue selon ses mérites littéraires ${ }^{(20)}$. Pour cette raison, bien qu'il affirme préférer les faits réels, étant donné " qu'il se dégage de notre vie passée une magie incomparable " $(A O, \mathrm{p}$. 103), Wang Er sélectionne avec soin les événements à inclure pour mieux répondre aux exigences de ses lecteurs :

Nous nous étions rendus coupables d' " erreurs graves ", nous méritions d'être fusillés. Nos dirigeants nous avaient accordé cette grâce immense de pouvoir confesser nos fautes. J'avais décidé, dans ces conditions, de nous peindre sous les traits les plus noirs. (AO, p. 104)

Le narrateur adopte ici la technique d'un complet détachement, point sur lequel l'autocritique fictionnelle écrite par Wang Er et le texte que le lecteur tient effectivement entre ses mains convergent. Le narrateur fait mine de prendre toutes les recommandations politiques au pied de la lettre, les poussant à leur point extrême d'absurdité et d'autocontradiction. Son usage des raisonnements répondant à une logique formelle (comme le rappellent les traducteurs anglais dans leur préface, le père de Wang Xiaobo était un logicien célèbre ${ }^{(21)}$ ) est particulièrement satirique. Lorsque Chen Qingyang lui demande pourquoi on l'appelle " chaussure trouée ", plutôt que de la défendre contre l'accusation en invoquant les faits, il entreprend de lui prouver, tout au long de son récit, que l'allégation est vraie, mettant ainsi les faits en accord avec les mots. De même, sommé d'avouer de « mauvaises » actions, il passe sous silence tout ce qui n'entre pas dans cette catégorie. Ainsi, la fiction finit par produire de la réalité : catégories politiques et slogans sont changés en événements par le respect scrupuleux de la logique formelle et par la vertu de la rédaction d'autocritiques. Le narrateur ajoute, dans l'un de ses apartés caractéristiques (souvent entre parenthèses) : «En réalité, cette sublime amitié n’était ni vraie ni fausse, comme toute chose en ce bas monde : si vous y croyez, elle s'avérera ; si vous en doutez, elle sera fausse " $(A O$, p. 36).

Cette attitude consistant à s'interroger sur l'entrelacement des faits et de la fiction, sur la façon dont les utopies se changent en faits historiques ne relève pas, chez Wang Xiaobo, d'une posture esthétique. Elle n'enlève rien à sa dénonciation de la Révolution culturelle, mais introduit plutôt une forme d'étonnement et de détachement. À un certain niveau, elle sert à montrer comment l'utopie politique de l'âge d'or crée un monde qu'on peut, par son extériorité aux règles de la logique et de la causalité, qualifier de fictionnel. C'est ce que le narrateur remarque quand Chen Qingyang, sur scène, est " luttée » : «Elle pensait, dans ces moments-là, qu' elle vivait dans un monde bien étrange " $\left(A O\right.$, p. 116) ${ }^{(22)}$. Plus profon-

20. De la même façon, un employé des ressources humaines dans l'université de Wang Er compare ensuite ses aveux à une œuvre littéraire $(A 0, \mathrm{p} .62)$.

21. Cf. Wang Xiaobo, Wang in Love and Bondage, trad. Hongling Zhang et Jason Sommer, Albany, SUNY Press, 2007

22. Cf. aussi : " Car, à quarante ans passés, elle considérait toujours le monde avec candeur. » $(A O$, p. 123) 
dément, le narrateur n'est pas intéressé par une déploration des " cicatrices "; même lors de sa confession, il réserve encore des piques acerbes contre les chefs, en se réappropriant leurs catégories et leurs façons de penser ; pareil en cela à Chen Qingyang, il crée un spectacle pour les chefs, tout en permettant aux lecteurs réels des années 1990, comme s'ils lisaient par-dessus l'épaule du commissaire, de partager la dérision dont celui-ci fait l'objet.

Dans cette perspective, l'âge d'or d'une sexualité débridée ne constitue pas tant une libération des contraintes sociales à la faveur des bouleversements de la Révolution culturelle, qu'une façon de se réapproprier l'expérience de la persécution. La technique par laquelle Chen Qingyang transforme la « réunion de lutte " en une représentation théâtrale trouve un écho dans la transformation par Wang Er de sa rééducation dans le Yunnan en " âge d'or " de libération sexuelle, grâce à l'écriture. L'autocritique rédigée par les deux personnages sert à refléter dans l'histoire elle-même cette réappropriation de l'expérience à travers l'écriture, voire à travers l'invention fictionnelle :

Tout le monde aimait lire les autocritiques que je composais. Au début, je n'écrivais qu'avec de grandes réticences. Mais plus je progressais dans mon récit, plus j'étais fasciné par mon sujet. Sans doute était-ce parce que tout ce que je relatais, je l'avais vе́сu $[. .$.$] . ( A O$, p. 102-103)

Tandis que la fuite hors de la ferme collective pour rejoindre la nature originelle du Yunnan est d'abord déclenchée par l'instinct de survie, elle se trouve progressivement réintégrée dans le discours de l' "âge d'or ", discours que, dans ce passage, Wang Er admet littéralement " réinventer " à travers un mélange complexe de confession et de fictionalisation. Le lecteur ne doit pas oublier le cadre dans lequel ce discours est produit, lié qu'il est à la rédaction - explicitement littéraire, voire fictionnelle - de l'autocritique de Wang $\mathrm{Er}^{(23)}$. En affirmant le caractère fictionnel et ludique de son écriture, Wang Er retrouve dans une certaine mesure une posture active, et centrée sur lui-même en tant que sujet de l'histoire plutôt que " victime " démunie dont les " cicatrices » devraient être rappelées en de larmoyantes remémorations des violences du maoïsme. De manière symptomatique, l'attitude de Wang Xiaobo à l'égard de l'âge d'or reste ambiguë ; néanmoins, elle ne doit pas être réduite à un discours foucaldien dans lequel toutes les formes de pouvoir auxquelles l'individu est soumis seraient équivalentes. Aussi la dernière section de cet article tente-t-elle de replacer les spécificités de la Révolution culturelle au sein d'un discours plus large de Wang Xiaobo sur les "trois âges ", ainsi que sur les contradictions entre l'individu et la société.

\section{Renoncement au monde ou entrée dans le monde}

Si le retour utopique de l'individu à une vie en pleine nature s'avère contraint et chargé de souffrances, la mise en fiction qui s'ensuit lui permet de réaffirmer sa subjectivité. Mais quel type de posture sociale implique cette forme minimale de préservation de l'autonomie individuelle? Dans quelle mesure ce conflit constitue-t-il une part inhérente de la condition humaine et dans quelle mesure est-il au contraire déterminé par le contexte spécifique de la Révolution culturelle ? Wang Er semble charger la métaphore de la castration du buffle d'un sens plus largement existentiel quand il remarque : "Je n'ai compris que bien plus tard que la vie était un lent processus de castration au maillet, qui fait vieillir les hommes et se dissiper jour après jour leurs folles espérances, pour qu'ils deviennent finalement semblables en tous points à des buffles castrés » $(A O$, p. 31$)$. Le thème revient en conclusion du récit lorsque Wang $\mathrm{Er}$, se retrouvant trente ans après avec Chen Qingyang dans la chambre d'hôtel, se rend compte qu'il éprouve encore des désirs sexuels : " Je me dépêchai donc de conclure ainsi de toute cette affaire : "À l'époque, on voulait vraiment notre peau, mais en définitive personne n'avait réussi à nous abattre" " $(A O$, 121). Le fait que Wang Er se soit ainsi préservé est-il le résultat de sa stratégie de retraite hors du monde?

Wendy Larson, dans un article récent, a proposé d'interpréter le récit comme une illustration, et dans le même temps comme une critique, à ses yeux imprégnée de taoisme, de la relation des intellectuels chinois au pouvoir. Pour elle, la vision du monde implicite de Wang Er est que l'individu, afin de se soustraire aux coups incessants de la politique, doit se retirer du monde, ou du moins ne jamais se confronter directement à lui. Considérant que les tendances anti-autoritaires de Wang Er sont secondaires par rapport à la sensibilité mé-

23. En outre, les récits des deux personnages ne coïncident pas, puisqu'un épisode crucial est omis par Wang Er (qui a conservé les copies carbones de ses autocritiques), alors que Chen Qingyang (qui a récupéré l'original des siennes au Yunnan) lui donne une importance décisive. II s'agit de deux claques données par Wang Er sur le derrière de Chen Qingyang lorsqu'il la porte sur le Mont Qingfeng. Dans la confession qu'elle écrit pour mettre fin à toutes les demandes ultérieures, Chen Qingyang écrit que c'est là le moment où « En un instant elle était tombée amoureuse de [Wang Er], pour toujours » $(A O$, p. 133), soulevant la question de savoir pourquoi Wang Er omet cet épisode. Alors que lui, dans ses aveux, dépeint l'« âge d'or » comme celui de l'éveil d'une sexualité libérée de toute forme de responsabilité sociale, Chen Qingyang charge cet « âge d'or » d'une signification tout à fait différente et " culturelle », le ramenant à des codes et conventions sociales (" tomber amoureux », " pour toujours »). Naturellement, cet écart ouvre la porte à d'innombrables interprétations de la nouvelle. 
tièrement asymétrique. Ainsi, la réunion du chapitre $9 \mathrm{du}-$ rant lequel Chen Qingyang est « luttée » excite sexuellement cette dernière, elle s'y comporte en victime consentante dans la mesure où elle se laisse ligoter, arracher les cheveux, et qu'elle prend plaisir à sa soumission : "Mais elle était heureuse et faisait tout ce qu'on lui disait de faire " $(A O, \mathrm{p}$. 117). Le fait d'être transportée ligotée dans la chambre d'hôtel parachève son excitation :

Dans ces moments-là, elle avait le visage empourpré comme si elle avait bu, et me disait : "Et si on entretenait notre vieille amitié ? Vite, je ne peux plus attendre! "Elle m'avoua que lorsque je la détachais, elle avait l'impression d'être un cadeau dont on défaisait l'emballage, et qu'alors elle était submergée par la joie. $(A O$, p. 117)

Après quoi, une scène ritualisée de sexe violent se déroule sur le bureau où Wang Er et Chen écrivent habituellement leur autocritique, la fenêtre ouverte sur des passants voyeurs qu'ils bombardent de poires dures comme des pierres. Or, toute cette scène se déroule comme la répétition et la conséquence naturelle du voyeurisme de la réunion de lutte, durant lequel Chen Qingyang avait déjà remarqué « que tous les hommes présents avaient la braguette saillante " $(A O$, p. 116) : elle suggère la dimension (plutôt que la nature) érotique du pouvoir et des persécutions, ainsi que l'étrange relation à la victime qu'il peut entraîner. Il est donc certain que Wang Xiaobo joue avec la double nature, à la fois transgressive et répressive, du maoïsme. Mais le lecteur peut-il vraiment croire que la Révolution culturelle et l'idéologie maoïste puissent se réduire à une variante de sado-masochisme dans laquelle les intellectuels chinois joueraient le rôle de victimes consentantes ou, au mieux, affirmeraient leur subjectivité à travers une forme opportuniste de plaisir individuel quills se ménageraient en renonçant à toute confrontation politique directe ?

Le débat sur la retraite hors du monde, bien que jamais formulé en termes taoïstes, structure d'une certaine façon le récit et donne sens à l'utopie d'une vie en pleine nature, telle qu'illustrée précédemment. Pour ce qui est de Wang Er, il aborde le sujet en posant la question de sa propre existence, par exemple au début, lorsqu'il fuit dans les montagnes après sa blessure au chapitre 3 :

Elle savait qu'en empruntant tel itinéraire on pouvait me rejoindre. C'était un fait indubitable, et pourtant elle avait de plus en plus tendance à en douter. Non, décidément, ce chemin ne devait mener nulle part, et Wang
Er ne se trouvait vraisemblablement pas dans la montagne. D'ailleurs, Wang Er existait-il ? (AO, p. 48)

Cette discussion montre que les fuites répétées de Wang Er dans les montagnes ne doivent pas simplement être vues comme un épisode au sein du récit : elles représentent la possibilité d'une retraite beaucoup plus radicale hors de l'existence. Si le chef d'équipe, et plus généralement le système administratif, ne reconnaissent pas ce qu'lls ne peuvent plus voir ("Wang Er? Qui c'est, Wang Er ? Connais pas »; $A O$, p. 51$)$, le narrateur croit quant à lui que cette forme de non-existence est elle-même illusoire :

Ainsi donc, il y avait des gens pour affirmer que Wang Er n'existait pas. C'est cela qui perturbait le plus les gens. Si une chose dont tout le monde affirme l'existence n'existe pas, c'est parce que toute réalité est illusoire. Mais certaines choses dont tout le monde nie l'existence existent nécessairement, par exemple Wang Er ; s'il n'avait pas existé, d'où serait venu ce nom? ( $A O$, p. 49 , traduction modifiée)

Ce passage contradictoire fait allusion au lieu commun bouddhiste sur la non-réalité de la réalité, qui sert à construire une série de paradoxes inextricables et trouve un écho dans des paralogismes qu'affectionne Wang Er ("Par exemple, les gens disaient que j'entretenais avec Chen Qingyang des relations illicites : c'était bien une preuve de mon existence "; $A O$, p. 52). Mais au cour de cette réfutation de la non-existence de Wang Er se trouve une prise de conscience : bien qu' un individu puisse "disparaitre " de la société, comme ce fut le cas pour les jeunes instruits envoyés à la campagne, il est pourtant impossible de choisir de ne plus exister, dans le sens d'une retraite complète du monde phénoménal (et donc politique). Bien que Wang Er désire quitter la société, par exemple après sa querelle silencieuse avec le député militaire, il finit par comprendre qu'un tel départ est impossible.

De la même façon, Chen Qingyang souhaite parfois se dissoudre dans la nature. Ainsi, la première fois qu'elle va rendre visite à Wang Er dans la montagne, "le cœur débordant de rêves fous " $(A O$, p. 125) :

Le ciel était très clair, il s'animait du vol des pigeons, et leurs roucoulements résonnaient au tréfonds d'ellemême. Elle désirait alors bavarder avec moi, de la même façon que jadis elle avait désiré s'unir avec le monde, se fondre dans l'univers. La pensée qu'elle aurait pu être seule au monde lui était insupportable. $(A O, p .126)$ 
À ce point du récit, elle caresse elle aussi brièvement l'idée de renoncer à toute préoccupation mondaine pour se fondre dans la nature. Cependant, au moment d'entrer dans la hutte et de voir la "laideur " du " petit bonze " de son compagnon, elle comprend aussi brusquement qu'il n'y a aucun moyen d'échapper aux relations de pouvoir qui structurent la société : «[...] elle avait poussé un cri de frayeur et tout espoir l'avait quittée " $(A O$, p. 124). Le sexe lui-même n'est donc pas seulement un objet de pure beauté naturelle et une sorte d'utopie, mais aussi une forme de soumission et d'aliénation pour Chen Qingyang (comme le montre le décalage entre leurs deux versions de l'épisode du Mont Qingfeng ${ }^{(25)}$ ). Dès lors, elle ne veut plus renoncer à l'existence mondaine :

C'était donc cela qu'on appelait la réalité ! Impossible de s'en débarrasser en s'éveillant, comme on le fait d'un rêve. En un instant elle comprit enfin de quoi le monde est fait, et l'instant d'après elle avait pris sa décision : elle poursuivrait son chemin, prête à affronter toutes les épreuves de la vie. Alors, son coeur déborda de joie. $(A O, 126)$

Ce passage renvoie à une autre justification de la fin de la vie montagnarde de Wang Er et Chen :

Bien sûr, la vie que nous avions menée dans les montagnes était elle aussi fascinante; mais à force [...], elle risquait de devenir lassante. C'est pourquoi elle voulut descendre en ville, endurer tous les malheurs engendrés par la société des hommes. $(A O$, p. 100)

Naturellement, la vraie raison du renoncement à l'utopie n'est pas seulement le fait qu'elle est lassante, mais, comme dans le premier passage, son impossibilité logique et son inexistence empirique. Les deux personnages en viennent à reconnaitre et à accepter les "tourments " du monde (renshi de cuican 人世的摧殘, jieshou cuican 接受摧殘) ${ }^{(26)}$ comme simples équivalents de la condition humaine. Il n'y a aucune alternative à la vie dans le monde et, si fictionnels et illogiques que les événements de la Révolution culturelle puissent paraitre, ils n'en sont pas moins la seule réalité dont on dispose.

La remarque sur l'impossibilité de se réveiller fait allusion à un épisode précédent dans lequel un grain de sable logé dans son œil fait tellement mal à Chen Qingyang qu'elle peut seulement espérer qu'il s'agit d'un mauvais rêve dont elle se réveillera :

C'était tellement insupportable qu'elle avait éclaté en sanglots, en espérant se réveiller en pleurant sur son petit lit, ce qui était chez elle une habitude profondément enracinée. Sangloter bruyamment pour pouvoir quitter un mauvais rêve et entrer dans un autre rêve, n'est-ce pas la folle espérance que nous caressons tous? ( $A O$, p. 125, traduction modifiée)

Mais s'il est naturel de nourrir de " folles espérances ", en particulier quand on est en présence d'une adversité politique comme celle que subissent les personnages du récit, ces espoirs finissent par s'envoler en face d'une réalité inébranlable :

Chen Qingyang me dit que l'homme était sur terre pour souffrir, jusqu'à ce qu'il meure. Une fois qu'on avait compris cette vérité, on pouvait tout endurer. (AO, p. 124)

L'humaine condition est souffrance, et l'on ne peut y échapper. Pour ces raisons, on doit être très prudent quand on interprète le récit de Wang Xiaobo comme l'évocation d'une utopie rurale. Alors même qu'il joue avec la dimension transgressive du maoisme, tournée en dérision et réduite trivialement à une sexualité sans entraves, le récit est structuré de telle sorte que les personnages prennent progressivement conscience qu'il n'est pas d'échappée hors des réalités de la condition humaine. En ce sens bien sûr, on peut dire que la Révolution culturelle ne fait que manifester cette souffrance intemporelle de l'humanité qui prend mille formes à travers la succession des temps et des lieux.

Pourtant, sous la plume de Wang Xiaobo, se confronter à la souffrance humaine n'est pas une expérience négative. Il n'encourage ni la retraite taoïste hors du monde, résultat de la désespérance des intellectuels après le désarroi et la résignation provoqués par le maoisme (ce que Dai Jinhua appellerait sans doute leur " masochisme »), ni ce que Wendy Larson appelle le laisser-faire, et qu'elle décrit comme un sentiment d'épuisement lié au passage du temps. Au contraire, Wang plaide pour un engagement actif dans le monde, pour un renoncement à toute forme d'utopie (politique, naturelle, sexuelle ou autre), et, finalement, pour la reconquête d'un rôle moteur pour le sujet dans l'histoire: Wang Er en donne l'exemple, en se réappropriant activement sa propre expérience par la narration. Le critique Chen Xiaoming, à propos d'un

25. Cf. ci-dessus la note 23 .

26. Le mot cuican a des connotations intéressantes : il est apparemment utilisé pour désigner la souffrance causée par les catastrophes naturelles, et est devenu fréquent pour évoquer la Révolution culturelle. Comme l'a suggéré un auditeur que nous remercions, il peut également s'agir d'une citation de la traduction de L'Amant de Marguerite Duras par Wang Daoqian. 
autre récit, décrit de la même façon le souci qu'a Wang de ramener ses personnages « à la culture ${ }^{(27)}$. » En ce sens, la contextualisation n'est pas utilisée pour relativiser ou diminuer la critique de la Révolution culturelle. Elle sert plutôt à dénoncer le tragique malentendu faisant du maoisme un âge d'or utopique, et qui a trop longtemps entravé son analyse et sa critique. Le ton adopté par Wang Xiaobo, fait d'ironie implacable et d'humour détaché, est à l'inverse destiné à dissiper les mirages de l'utopie, et à encourager l'engagement rationnel des individus dans la société, ainsi que le maintien de leurs facultés critiques. Wang Xiaobo élabore son œuvre à partir d'un cliché de la littérature des " jeunes instruits ", l'éveil sentimental des jeunes gens à la campagne. S'il l'investit d'une dimension explicitement sexuelle, il le situe en même temps à des niveaux philosophiques et politiques jamais atteints jusqu'alors. Bien qu'il y ait certainement un aspect psychologiquement libérateur dans l'épisode historique quil dépeint, le fait de le présenter comme un " âge d'or " est avant tout hautement ironique : il s'agit de démasquer dans l'expression un euphémisme qui masque une configuration politique dans laquelle la fiction domine la réalité, et où la quête d'une pureté révolutionnaire est simplement une stratégie pour humilier autrui et satisfaire les appétits des commissaires politiques au pouvoir. Ces réflexions culminent dans la prise de conscience du caractère illusoire de toute retraite néotaoiste hors du monde de la politique maoiste : Chen et Wang, à l'issue du récit, aspirent à retourner dans la société, et à retrouver les " tourments du monde ". Cela signifie-t-il quils ont effectivement été lentement " castrés " par la société et intimidés au point d'accepter la souffrance comme un donné de l'existence humaine ? Il nous semble qu'accepter l'existence de la souffrance comme une constante anthropologique ne conduit pas à excuser ses causes dans un contexte politique particulier. Accepter l'entrée dans les « tourments du monde ", plutôt que de nier l'existence de ce dernier et de rechercher hors de lui la réalisation d'utopies politiques, constitue probablement déjà une avancée par rapport à la constellation politique dysfonctionnelle qui a caractérisé la modernité politique chinoise.

Qu'est-ce qui, dès lors, a rendu ce bref récit si séduisant aux yeux des lecteurs chinois ? Au-delà du scandale, mais également des tendances intellectuelles post-modernes et d'une approche uniquement sexuelle des luttes politiques (sans doute inspirée par certaines lectures de Foucault), Wang Xiaobo marque une rupture radicale, non pas tant avec une " tradition " de soumission des intellectuels chinois « masochistes ", qu'avec l'inertie de la société civile face au maoïsme - laquelle n'a pu se représenter autrement qu'en victime ou, au mieux, comme réinventant un espace privé en se retirant du monde. Wang Er, l'indomptable héros de Wang Xiaobo, d'une part démystifie les persécutions de la Révolution culturelle en recourant à l'humour, dans la veine du héros d'Imre Kertész dans Etre sans destin ou de l'Oskar de Günter Grass ; d'autre part, il incarne une foi renouvelée dans l'écriture, laquelle ne sert pas seulement à rappeler des crimes et à ménager une place à la subjectivité, mais aussi à recréer un signification collective.

En dernière analyse, le succès incomparable des écrits de Wang dans les années 1990 est donc sans doute lié à l'expérience libératrice d'un irrespect total, non seulement à l'égard des idéaux périmés du maoïsme (déjà discrédités par les écrivains liumang des années 1980), mais aussi à l'égard de la sacralisation des souffrances des victimes de la Révolution culturelle. Cette génération, qui à cette époque approchait de la vieillesse, a bien souvent utilisé (à quelques exceptions près) son rejet (légitime) du maoisme, pour prêcher l'acceptation des compromis de Deng Xiaoping, surtout après $1989^{(28)}$. Le succès de Wang Xiaobo témoigne de l'existence d'un point de vue critique concernant l'idée largement répandue qu'il faudrait accepter le présent parce que le passé était pire. Tandis que pour les historiens de la Révolution culturelle, les positions de Wang Xiaobo peuvent sembler excessivement audacieuses et iconoclastes, dans un contexte où les bases historiographiques ne sont pas encore stabilisées (dans le cas de l'Holocauste par exemple, ce genre de critique des victimes n'est devenu possible qu'une fois les faits historiques établis de façon certaine), elles constituent aussi une provocation ouvrant un espace bienvenu et très nécessaire à un débat sur l'histoire récente de la Chine. •

\section{- Traduit par Guillaume Dutournier}

27. «Le questionnement de Wang Xiaobo sur l'existence individuelle est entièrement nu. On accorde généralement une valeur ajoutée à son œuvre parce qu'elle aborde le sexe mais Wang le réduit à sa plus pure configuration. Foucault écrit que le sexe et le pouvoir sont les deux faces d'une même pièce. Dans Wode yinyang liangjie, xiao Sun représente la culture ; elle entre dans les profondeurs cachées de Wang Er afin de le guérir. Mais après l'avoir guéri de son impotence et en avoir fait un vrai homme, elle le ramène en même temps à la culture. ", in Ai Xiaoming, Li Yinhe (éd.), Langman qishi (Le Chevalier romantique), Pékin, Zhongguo qingnian, 1997, p. 262.

28. La littérature des cicatrices et des films comme Le Cerf-Volant bleu de Tian Zhuangzhuang (1993) sont sans doute des exemples de la manière dont la sacralisation des souffrances des victimes peut finir par entraver le changement politique. Cet usage souligne l'ambiguïté inhérente au postulat de l'incommensurabilité de la souffrance humaine (« les générations suivantes ne peuvent pas comprendre »), qui sous-tend la littérature des cicatrices. 